\title{
Impact of COVID-19 pandemic on outpatient appointments of rheumatic patients in a non-outbreak area of China
}

\author{
Tianhua Xie - Dong Wang · Xia Wang - Qingrui Yang · Hongsheng Sun · Ruihong Liu - Ming Li (D)
}

Received: 16 November 2020 / Accepted: 5 May 2021 / Published online: 18 June 2021

(C) Springer-Verlag GmbH Austria, part of Springer Nature 2021

\begin{abstract}
Summary
Background Coronavirus disease 2019 (COVID-19) infection has caused huge impacts on all of people's lives and health systems. In response to the COVID-19 pandemic, China was the first country to impose lockdown. We aimed to study the influence of COVID19 on the outpatient visits of rheumatic patients in a non-outbreak area of China.

Methods We selected three provincial or ministerial hospitals in Jinan, and collected the outpatient appointments data in rheumatology and immunology departments during the Shandong Province first-level public health emergency response period from 25 January 2020 to 8 March 2020.

Results In the early stage, the number of outpatient appointments in the rheumatology and immunology
\end{abstract}

Data Availability Statement The data that support the findings of this study are available from the corresponding author, $\mathrm{M}$. Li, upon reasonable request. The corresponding author had full access to all the data in the study and had final responsibility for the decision to submit for publication.

T. Xie, MD $\cdot$ X. Wang, MD $\cdot$ Q. Yang, MD $\cdot$ H. Sun, MD .

M. Li, MD ( $ه)$

Department of Rheumatology and Immunology, Shandong

Provincial Hospital Affiliated to Shandong First Medical University (Shandong Provincial Hospital), Jinan, China 272951743@qq.com

\section{Wang, MD}

Department of Medical Administration, Shandong Provincial Hospital Affiliated to Shandong First Medical University (Shandong Provincial Hospital), Jinan, China

\section{R. Liu, MD (ه)}

Department of Statistics and Medical Record Management, Shandong Provincial Hospital Affiliated to Shandong First Medical University (Shandong Provincial Hospital), Jinan, China

939350140@qq.com departments of the three provincial or ministerial hospitals were significantly reduced, and gradually restored in the late stage. It showed that in the face of major infectious diseases, strict quarantine measures with the cooperation of the public not only controls the epidemic in a short time, but also lifts the quarantine measures and opens general outpatient clinics in hospitals as soon as possible, thus minimizing the impact on other patients.

Interpretation The impact on the western hospital was greater than that on the Chinese medicine hospital, and the impact on the back-up designated hospitals for COVID-19 was the greatest. Online appointment can reduce the risk of infection in outpatients, but not completely solve the follow-up problem of rheumatic patients. Telemedicine provides a new solution for both management of rheumatic patients and control of COVID-19.

Keywords Backup designated hospital · Traditional Chinese Medicine $\cdot$ First-level public $\cdot$ Health emergency response $\cdot$ Telemedicine $\cdot$ Chronic disease

\section{Keypoints}

The study indirectly proved the importance of quarantine measures during the COVID-19 epidemic period.

The study also reflected the lack of online appointments during the COVID-19 epidemic period.

\section{Introduction}

In late December 2019, cases of pneumonia of unknown etiology were found in Wuhan, Hubei Province, China [1]. After analyzing the whole genome sequence of respiratory tract virus, the pathogen was proved to be a novel coronavirus. The Coronavirus Study Group (CSG) of the International Commit- 
Table 1 Outpatient appointments of rheumatology and immunology departments in the three provincial and ministerial hospitals from 2017 to 2020 - Changes of outpatient appointments over year

\begin{tabular}{|l|l|l|l|l|l|l|}
\hline & SPH & \multicolumn{2}{c}{ QH of SDU } & \multicolumn{3}{l}{ AH of SDUTCM } \\
\hline Year & Person-time & Sequential growth rate (\%) & Person-time & Sequential growth rate (\%) & Person-time & Sequential growth rate (\%) \\
\hline 2017 & 2335 & NA & 1063 & NA & 1400 & NA \\
\hline 2018 & 3254 & 39.36 & 2224 & 109.22 & 3062 & 118.71 \\
\hline 2019 & 4012 & 23.29 & 3266 & 46.85 & 3733 & 21.91 \\
\hline 2020 & 1205 & -69.97 & 976 & -70.12 & 1969 & -47.25 \\
\hline Total & 10,806 & NA & 7529 & NA & 10,164 & NA \\
\hline
\end{tabular}

SPH Shandong Provincial Hospital, QH of SDU Qilu Hospital of Shandong University, AH of SDUTCM Affiliated Hospital of Shandong University of Traditional Chinese Medicine

tee on Taxonomy of Viruses assessed the novelty of the human pathogen tentatively named 2019 novel coronavirus (2019-nCoV), and formally designated it as severe acute respiratory syndrome coronavirus 2 (SARS-CoV-2). The disease caused by SARS-CoV-2 was named coronavirus disease 19 (COVID-19) by the World Health Organization, which declared that the global coronavirus crisis was a pandemic on 11 March 2020 [2]. Up to 28 June 2020 the spread of the SARS-CoV-2 in 212 countries and regions had resulted in 10,001,527 confirmed cases and 499,124 deaths, with a mortality rate of $4.99 \%$ [3]. The COVID19 has caused huge impacts on all of people's lives and healthcare systems. There is no specific cure method for the disease, although several kinds of vaccines are meanwhile available [4].

In response to the COVID-19 pandemic, China adopted a series of measures. According to the Law of the People's Republic of China on Prevention and Treatment of Infectious Diseases and the Regulation on Responses to Public Health Emergencies, China was the first country to impose lockdown. From 23 January 2020, all provinces, autonomous regions and municipalities in China activated first-level public health emergency response (hereinafter referred to as first-level response) for preventing, controlling and stopping infections with COVID-19 in accordance with the emergency preplans. With the support and cooperation of 1.4 billion Chinese people, the epidemic was quickly under control [5]. Since 3 March 2020, the national daily increment has reduced to about 100 cases, and the quarantine measures have been gradually lifted. Since 8 March 2020 (44 days after first-level response), Shandong province has lowered its public health emergency response to second level. Since 8 April 2020, the lockdown in Wuhan, the city suffered the hardest hit by SARS-CoV-2 in China, has loosened. As of 28 June 2020, 83,512 confirmed cases and 4634 deaths with a mortality rate of $5.55 \%$ have been reported by the national authorities in China [3]. In a bid to contain the contagion, Italy was one of the first European countries to impose nationwide restrictions in March [6]; however, the lack of scientific knowledge about SARS-CoV-2 and as a tourist country [7], there are still more than 700 new confirmed cases a day and the quarantine cannot be lifted in Italy. Moreover, Italy has the largest older population in Europe, nearly a quarter (23.3\%) of the population aged $\geq 65$ years, and with a median age (47.3 years) ranking second in the world [8], which increases the difficulty of COVID-19 pandemic control. As of 28 June 2020, 240,136 confirmed cases and 34,716 deaths with a mortality rate of $14.46 \%$ have been reported in Italy [3]. Most countries have imposed severe restrictions in recent months trying to slow the spread of the SARS-CoV-2; however, there is one country standing out from the crowd: Sweden. In Sweden, there is no lockdown or strict social distancing policy. Most schools, restaurants and companies are still open, only basic hygiene rules will continue to apply [9]. As of 28 June 2020, 65,137 confirmed cases and 5280 deaths with a mortality rate of $8.11 \%$ have been reported in Sweden. The Single day mortality rate gradually rose to be the first in Europe [3]. The COVID-19 pandemic has resulted in unprecedented challenges to healthcare worldwide. In outbreak areas, most medical resources are used to treat COVID-19 patients [10]. The increased number of COVID-19 patients has overwhelmed healthcare systems in many countries. Medical staff are either infected or redistributed, which significantly affects the standard care for patients with emergency, cancer, elective surgery and chronic diseases [11-15]. In non-outbreak areas, the government took a series of prevention and control measures to stop the infection of COVID-19. Patients with chronic diseases such as rheumatism, were restricted to hospitals, even they needed regular outpatient follow-up [16]. A rheumatism questionnaire survey in the USA showed that most rheumatic patients were affected by the COVID19 epidemic [17].

In order to study the impact of COVID-19 on outpatient appointments of rheumatic patients in a nonoutbreak area of China, we selected three provincial or ministerial hospitals in Jinan, collected the outpatient appointments data in rheumatology and immunology department during the Shandong Province first-level response period from 25 January 2020 to 8 March 2020, and compared the outpatient appointments data with the same period in previous years. 
Fig. 1 Outpatient appointimmunology departments in the three provincial and ministerial hospitals from 2017 to 2020 -Changes of outpatient appointments over day in every year, a 2017, b 2018, c 2019, d 2020. The horizontal axis shows a total of 44 days Eve. The vertical axis shows outpatient appointments of rheumatology and immunology departments per day. SPH Shandong Provincial Hospital, $Q H$ of SDU Qilu Hospital of Shandong University, $A H$ of SDUTCM Affiliated Hospital of Shandong University of Traditional Chinese Medicine ments of rheumatology and since Chinese New Year's
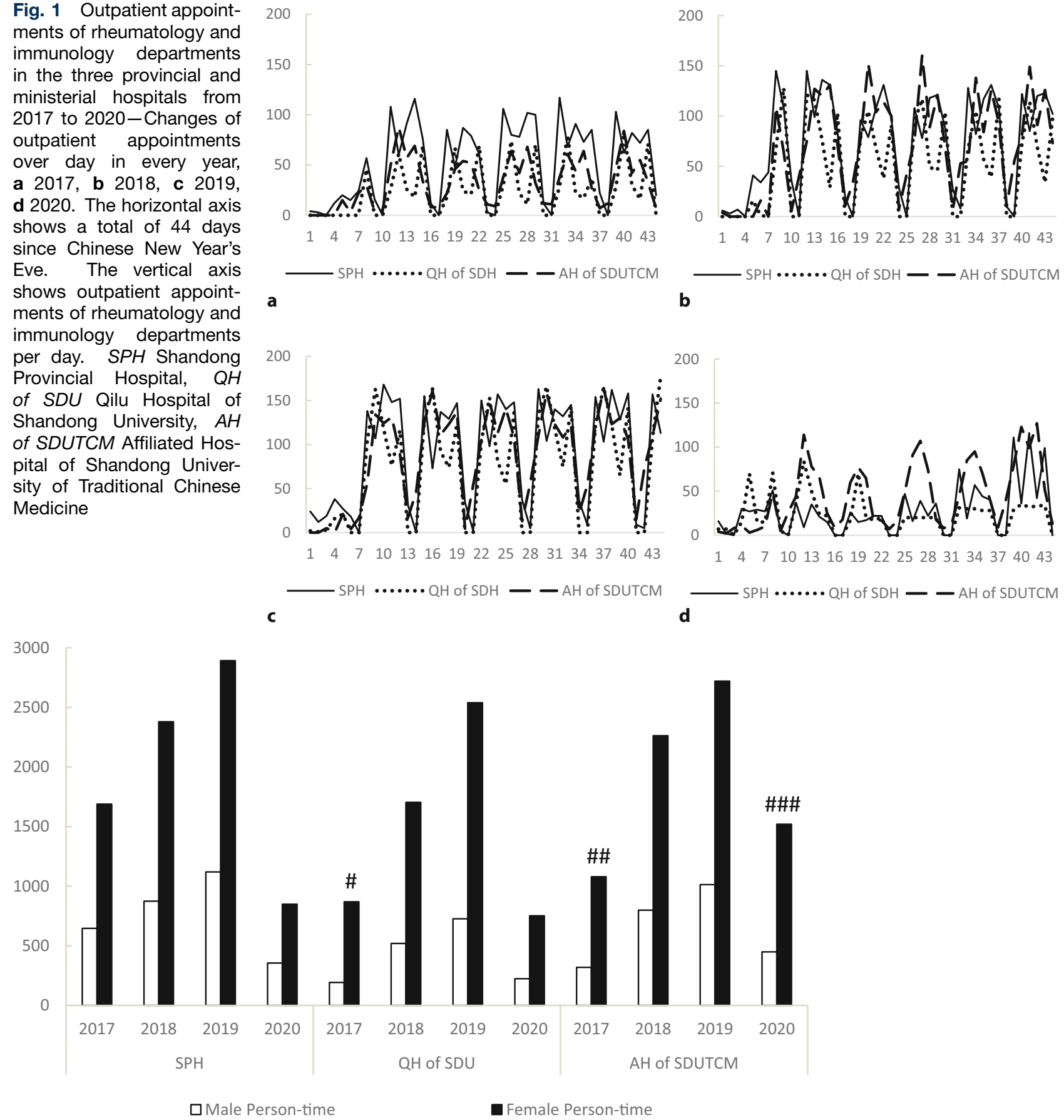

Fig. 2 Gender comparison of outpatient appointments of rheumatology and immunology departments in the three provincial and ministerial hospitals from 2017 to 2020. SPH Shandong Provincial Hospital, QH of SDU Qilu Hospital of Shandong University, AH of SDUTCM Affiliated Hospital of Shandong University of Traditional Chinese Medicine. \# Compared with the same hospital in $2018, x^{2}=11.5602$,
$P=0.0007$; compared with the same hospital in 2019 , $\mathrm{x}^{2}=8.0691, P=0.0045$; compared with the same hospital in $2020, x^{2}=7.19, P=0.0073$. \#\# Compared with the same hospital in 2018, $\mathrm{x}^{2}=5.3575, P=0.0206$; compared with the same hospital in 2019, $x^{2}=9.6977, P=0.0018$. \#\#\# Compared with the same hospital in 2018, $\mathrm{x}^{2}=6.9567, P=0.0084$; compared with the same hospital in $2019, \mathrm{x}^{2}=12.6933, P=0.0004$ 
Table 2 Comparison of gender composition of outpatient appointments among the three provincial and ministerial hospitals from 2017 to 2020

\begin{tabular}{|c|c|c|c|c|c|c|c|c|}
\hline & 2017 & & 2018 & & 2019 & & 2020 & \\
\hline & $x^{2}$ value & $P$ value & $x^{2}$ value & $P$ value & $x^{2}$ value & $P$ value & $x^{2}$ value & $P$ value \\
\hline SPH to QH of SDU & 35.5266 & $<0.0001$ & 8.5686 & 0.0034 & 30.4197 & $<0.0001$ & 12.0062 & 0.0005 \\
\hline SPH to AH of SDUTCM & 10.5555 & 0.0012 & 0.513 & 0.4738 & 0.5894 & 0.4427 & 17.9396 & $<0.0001$ \\
\hline $\mathrm{QH}$ of SDU to AH of SDUTCM & 8.0975 & 0.0044 & 5.0627 & 0.0244 & 22.1774 & $<0.0001$ & 0.008 & 0.9286 \\
\hline
\end{tabular}

\section{Material and methods}

\section{Clinical data}

From three provincial or ministerial hospitals in Jinan, Shandong Provincial Hospital Affiliated to Shandong First Medical University (hereinafter referred to as Shandong Provincial Hospital) as a back-up designated hospital, Qilu Hospital of Shandong University (hereinafter referred to as Qilu Hospital) as a non-designated hospital, and Affiliated Hospital of Shandong University of Traditional Chinese Medicine (hereinafter referred to as Affiliated Hospital of SDUTCM) as a non-designated hospital, outpatient appointments data of rheumatology and immunology department were collected during the first level period of Shandong Province in 2020 (a total of 44 days since Chinese New Years Eve), and the same period in 2017, 2018 and 2019. The information included the number of patients, gender, age, and region. Since the study is not included in the biomedical research activities of the "Measures for the Ethical Review of Biomedical Research Involving Humans" formulated by the National Health Commission of P.R. China, it is not suitable for conducting ethical reviews and complying with the principle of informed consent.

\section{Statistical methods}

The data were analyzed with SPSS 20.0 software (IBM, Armonk, NY, USA). Descriptive statistics included mean, rated, composition ratio etc. Single factor analysis was conducted by $\chi^{2}$-test. The difference was statistically significant with $P<0.01$. A prediction model for rheumatic outpatient appointments was established by negative binomial regression, and the test level was set as 0.05 . When composition ratios of multiple samples were compared pairwise, the test level was adjusted as $\alpha^{\prime}=1-\sqrt[m]{1-\alpha}, m=C_{k}^{2}, \alpha^{\prime}$ is adjusted test level, $\alpha$ is test level, $m$ is the number of comparisons, and $k$ was the number of groups.

\section{Patient and public involvement}

Patients or the public were not involved in the design, or conduct, or reporting, or dissemination plans of our research.

\section{Results}

\section{Changes in number of outpatient appointments}

From 2017 to 2019, the number of outpatient appointments for rheumatology and immunology department in each hospital showed a yearly increasing trend. In 2020, the number of outpatient appointments all decreased significantly during the first-level response period of Shandong Province, and the reduction in Shandong Provincial Hospital and Qilu Hospital was greater than that in Affiliated Hospital of SDUTCM (Table 1).

Every year from 2017 to 2019, the number of outpatient appointments in each hospital resumed after the Spring Festival holiday. Before 17 February 2020, in the early stage of Shandong Province's first-level response, the number of outpatient appointments in Shandong Provincial Hospital was significantly affected in the first 24 days since Chinese New Years Eve, while those the numbers in Qilu Hospital and Affiliated Hospital of SDUTCM were less affected. Since 2 March 2020, 39 days after Chinese New Years Eve, the numbers of outpatient appointments in Shandong Provincial Hospital and Affiliated Hospital of SDUTCM started to rise, and that in Qilu Hospital had not yet rebounded. The first-level response measures had a greater impact on the number of outpatient appointments in Shandong Provincial Hospital and Qilu Hospital than that in Affiliated Hospital of SDUTCM (Fig. 1).

\section{Gender comparison of outpatient appointments}

In the three hospitals, the majority of outpatient appointments in every year were female. In Shandong Provincial Hospital, there was no statistical difference in gender comparison in every year. In Qilu Hospital, the proportion of female patients was higher in 2017 than those in other years, and the difference was statistically significant. In Affiliated Hospital of SDUTCM, the proportion of female patients in 2017 was higher than those in 2018 and 2019, the proportion of female patients in 2020 was higher than those in 2018 and 2019, and the difference was statistically significant, which means that the proportion of female outpatients in Affiliated Hospital of SDUTCM increased during the first-level response of Shandong 


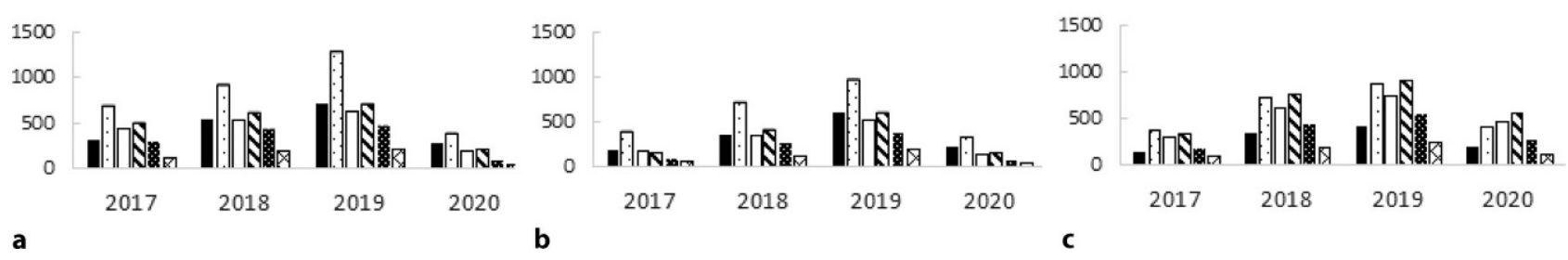

Fig. 3 Age structure (in years) of outpatient appointments of rheumatology and immunology departments in the three provincial and ministerial hospitals from 2017 to 2020 . a SPH, b QH of SDU, c AH of SDUTCM. SPH Shandong Provincial

Province (Fig. 2). In every year, the proportion of female outpatient appointments in Shandong Provincial Hospital was lower than that in Qilu Hospital. In 2017 and 2020, the proportion of female outpatient appointments in Shandong Provincial Hospital was lower than that in Affiliated Hospital of SDUTCM. Except for 2020, the proportion of female outpatient appointments in Affiliated Hospital of SDUTCM was lower than that in Qilu Hospital of Shandong (Table 2).

\section{Age comparison of outpatient appointments}

In Shandong Provincial Hospital and Qilu Hospital, the largest composition ratio of outpatient appointments was the $30-40$ years age group every year. In Affiliated Hospital of SDUTCM, the proportion of outpatient appointments aged 30-40 years was the highest in 2017, while the proportion of outpatient appointments aged 50-60 years was the highest in 2018, 2019 and 2020. The first-level response measures in Shandong province did not affect the age composition ratio of outpatient appointments in all the three hospitals (Fig. 3).

Comparing Shandong Provincial Hospital with Qilu Hospital, there were statistically significant differences in the composition ratio of outpatient appointments aged under 30, 30-40, 50-60 and 60-70 years in 2017. There was statistically significant difference in the composition ratio of outpatient appointments aged $30-40$ years in 2018 , while there was no statistically significant difference in the composition ratio of all age groups in 2019 and 2020.

Comparing Shandong Provincial Hospital with Affiliated Hospital of SDUTCM, the differences in composition ratios of outpatient appointments in 2017 were statistically significant in the age group of under 30 and above 70 years old. The differences in composition ratios of outpatient appointments in 2018 were statistically significant in the age group of under 30 , $30-40$, and 40-50 years old. The differences in composition ratios of outpatient appointments in 2019 were statistically significant in all age groups. The differences in composition ratios of outpatient appointments in 2020 were statistically significant in all age groups except for above 70 years old group.
Hospital, QH of SDU Qilu Hospital of Shandong University, $A H$ of SDUTCM Affiliated Hospital of Shandong University of Traditional Chinese Medicine

Comparing Affiliated Hospital of SDUTCM with Qilu Hospital, there were statistically significant differences in the composition ratios of outpatient appointments in the age groups except for above 70 years old group from 2017 to 2020. Among the three hospitals, Affiliated Hospital of SDUTCM had the most outpatient appointments for the older people. With the increase of years, there was no difference in the age composition ratio between Shandong Provincial Hospital and Qilu Hospital (Table 3).

\section{Geographical distribution of outpatient appointments}

In the three hospitals, the proportion of Jinan outpatient appointments in 2020 was higher than those in other years. In Shandong Provincial Hospital, the proportion of Jinan outpatient appointments in 2019 was higher than those in 2017 and 2018. In Qilu Hospital, the proportion of Jinan outpatient appointments in 2019 was lower than in 2017. In Affiliated Hospital of SDUTCM, the proportion of Jinan outpatient appointments in 2019 was higher than that in 2017. All the above differences were statistically significant, which means that the first-level response measures in Shandong Province had affected the geographical sources of outpatients in the three hospitals (Fig. 4).

In every year, the proportions of Jinan outpatient appointments in Shandong Provincial Hospital and Qilu Hospital were lower than that in Affiliated Hospital of SDUTCM. In 2017 and 2018, the proportion of Jinan outpatient appointments in Shandong Provincial Hospital was lower than that in Qilu Hospital. All the above differences were statistically significant (Table 4).

Overall, the outpatient appointments of Shandong Provincial Hospital and Qilu Hospital were more geographically distributed than Affiliated Hospital of SDUTCM, and there was little difference in the geographical distribution between Shandong Provincial Hospital and Qilu Hospital.

\section{Analysis of negative binomial regression model}

A negative binomial regression model was established by using the number of outpatient appointments in 
Table 3 Age structure of outpatient appointments of rheumatology and immunology departments in the three provincial and ministerial hospitals from 2017 to 2020

\begin{tabular}{|c|c|c|c|c|c|c|c|c|c|c|c|c|}
\hline & \multicolumn{2}{|c|}{$<30$ years } & \multicolumn{2}{|c|}{$30-40$ years } & \multicolumn{2}{|c|}{$40-50$ years } & \multicolumn{2}{|c|}{$50-60$ years } & \multicolumn{2}{|c|}{$60-70$ years } & \multicolumn{2}{|c|}{$>70$ years } \\
\hline & $x^{2}$ value & $P$ value & $x^{2}$ value & $P$ value & $x^{2}$ value & $P$ value & $x^{2}$ value & $P$ value & $x^{2}$ value & $P$ value & $x^{2}$ value & $P$ value \\
\hline \multicolumn{13}{|l|}{2017} \\
\hline $\mathrm{SPH}$ to $\mathrm{QH}$ of $\mathrm{SDU}$ & 7.7218 & 0.0055 & 23.365 & $<0.0001$ & 3.691 & 0.0547 & 16.1952 & $<0.0001$ & 17.4667 & $<0.0001$ & 3.4976 & 0.0615 \\
\hline SPH to AH of SDUTCM & 8.6519 & 0.0033 & 3.7273 & 0.0535 & 2.794 & 0.0946 & 3.2566 & 0.0711 & 0.0724 & 0.7878 & 4.9112 & 0.0267 \\
\hline $\begin{array}{l}\text { QH of SDU to AH of } \\
\text { SDUTCM }\end{array}$ & 25.068 & $<0.0001$ & 35.7523 & $<0.0001$ & 9.745 & 0.0018 & 26.6488 & $<0.0001$ & 13.4998 & 0.0002 & 0.0227 & 0.8804 \\
\hline \multicolumn{13}{|l|}{2018} \\
\hline $\mathrm{SPH}$ to $\mathrm{QH}$ of $\mathrm{SDU}$ & 0.7654 & 0.3817 & 9.8617 & 0.0017 & 0.873 & 0.3501 & 0.4178 & 0.518 & 2.4466 & 0.1178 & 0.0001 & 0.9921 \\
\hline SPH to AH of SDUTCM & 47.056 & $<0.0001$ & 17.381 & $<0.0001$ & 12.18 & 0.0005 & 31.0116 & $<0.0001$ & 0.9358 & 0.3334 & 0.7725 & 0.3795 \\
\hline $\begin{array}{l}\text { QH of SDU to AH of } \\
\text { SDUTCM }\end{array}$ & 29.515 & $<0.0001$ & 47.6048 & $<0.0001$ & 16.33 & $<0.0001$ & 31.4997 & $<0.0001$ & 5.8081 & 0.016 & 0.6108 & 0.4345 \\
\hline \multicolumn{13}{|l|}{2019} \\
\hline SPH to $\mathrm{QH}$ of $\mathrm{SDU}$ & 0.6309 & 0.427 & 3.7376 & 0.0532 & 0.114 & 0.7352 & 0.3868 & 0.543 & 0.024 & 0.8768 & 0.583 & 0.4451 \\
\hline SPH to AH of SDUTCM & 70.898 & $<0.0001$ & 67.0036 & $<0.0001$ & 22.54 & $<0.0001$ & 55.7341 & $<0.0001$ & 13.8963 & 0.0002 & 4.5896 & 0.0322 \\
\hline $\begin{array}{l}\text { QH of SDU to AH of } \\
\text { SDUTCM }\end{array}$ & 78.297 & $<0.0001$ & 34.857 & $<0.0001$ & 17.27 & $<0.0001$ & 41.4913 & $<0.0001$ & 11.3597 & 0.0008 & 1.6372 & 0.2007 \\
\hline \multicolumn{13}{|l|}{2020} \\
\hline $\mathrm{SPH}$ to $\mathrm{QH}$ of SDU & 0.1142 & 0.7355 & 0.6126 & 0.4338 & 2.752 & 0.0971 & 0.344 & 0.5575 & 1.0534 & 0.3047 & 0.0153 & 0.9015 \\
\hline SPH to AH of SDUTCM & 107.86 & $<0.0001$ & 54.785 & $<0.0001$ & 22.75 & $<0.0001$ & 44.2755 & $<0.0001$ & 31.984 & $<0.0001$ & 1.5245 & 0.2169 \\
\hline $\begin{array}{l}\text { QH of SDU to AH of } \\
\text { SDUTCM }\end{array}$ & 105.78 & $<0.0001$ & 61.6769 & $<0.0001$ & 37.51 & $<0.0001$ & 45.8234 & $<0.0001$ & 17.7818 & $<0.0001$ & 1.0341 & 0.3092 \\
\hline
\end{tabular}

rheumatology and immunology department of three hospitals from 2017 to 2019 as the dependent variable, and the year, the number of days from Chinese New Year's Eve, the week and the medical institutions as the independent variables. The fitting results were shown as model AIC3759.587 (akaike information criterion), BIC3787.296 (bayesian information criterion) in Table 5.

Under the same conditions of other factors, the number of outpatient appointments for each additional year increased by $54.36 \%$, the number of outpatient appointments for each additional day from Chinese New Year's Eve increased by $2.71 \%$, and the number of outpatient appointments for each additional day decreased by $28.67 \%$ from Monday. Compared with Shandong Provincial Hospital, Qilu Hospital had 40.73\% lower outpatient appointments, and Affiliated Hospital of SDUTCM had $13.71 \%$ lower outpatient appointments.

Comparing the predicted number of outpatient appointments with the actual number of outpatient appointments, it can be seen from Table 6 that the impact on the three hospitals during the first-level response period in Shandong Province, from large to small, was Shandong Provincial Hospital, Qilu Hospital, and Affiliated Hospital of SDUTCM.

\section{Discussion}

Our study showed that during the non-epidemic period, the number of outpatient appointments in the rheumatology and immunology department of the three hospitals from Jinan showed an increasing trend year by year, and the majority of the patients were female, mainly aged 30-60 years. In the early stage of Shandong Province's first-level response, the lockdown policy was conducive to the control of COVID19 , and the number of outpatient appointments in the three hospitals decreased significantly. The degree of impact in descending order was Shandong Provincial Hospitals as a back-up designated hospital, Qilu Hospital as a non-designated hospital, and Affiliated Hospital of SDUTCM as a non-designated hospital. In the late stage of Shandong Province's first-level response, the number of outpatient appointments gradually picked up.

Although it is a non-outbreak area, Shandong Province has a resident population of more than 100 million and a large mobile population due to Spring Festival holiday, the prevention and control of COVID-19 epidemic is not optimistic. Even on the special day of the Chinese New Year's Eve, 24 January 2020, the Shandong Provincial Government still took the lead in the country to activate first-level public health emergency response, and adopted measures such as restricting public transportation, remaining under closed-off management community control to prevent infection risks of COVID-19, establishing designated hospitals and backup designated hospitals to treat COVID-19 patients, and deploying medical staff to open fever clinics and support the anti-epidemic work in Hubei Province. The above measures were 


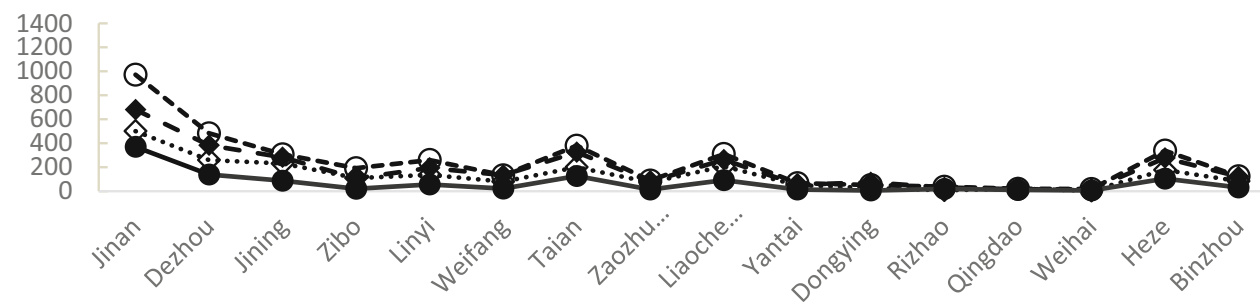

a

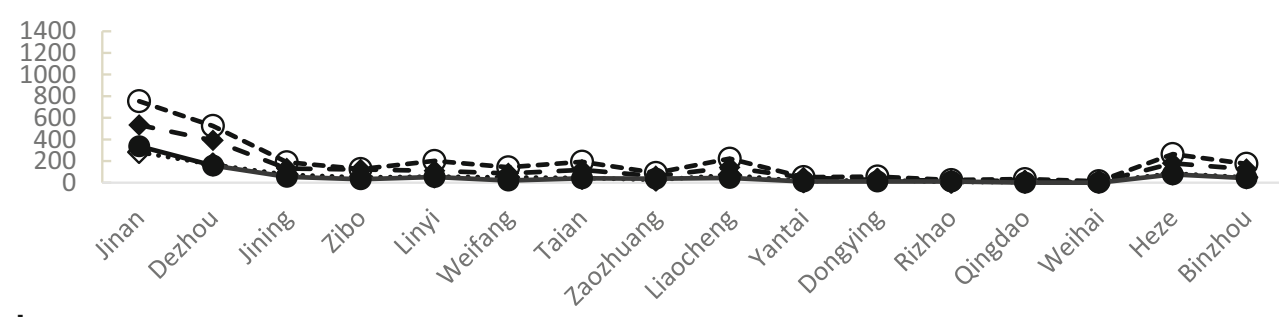

b

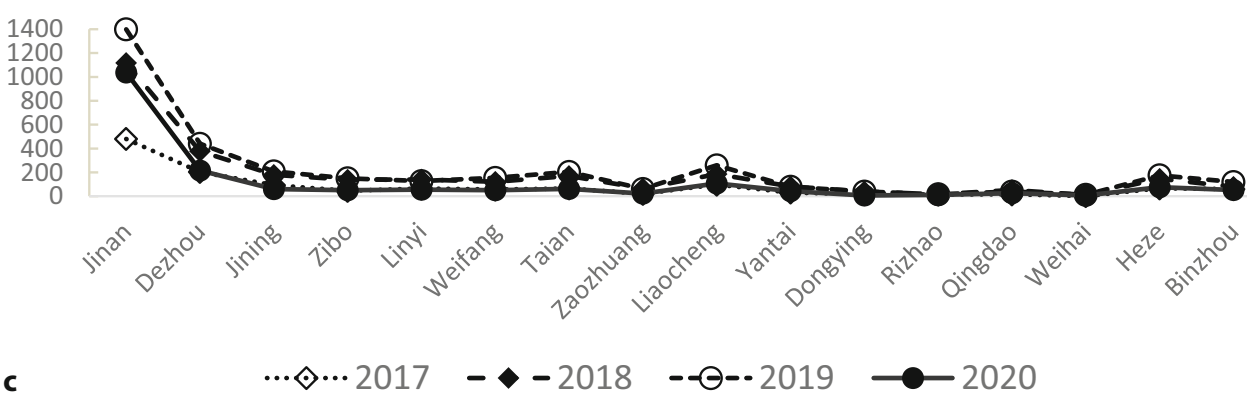

Fig. 4 Geographical distribution of outpatient appointments of rheumatology and immunology departments in the three provincial and ministerial hospitals from 2017 to 2020. a SPH-From Jinan of 2019 , compared with that of $2018, x^{2}=10.1973, P=0.0014$; compared with that of 2017, $x^{2}=5.099, P=0.0239$. From Jinan of 2020 , compared with that of $2019, x^{2}=22.8803, P<0.0001$; compared with that of 2018, $x^{2}=48.6613, P<0.0001$; compared with that of $2017, x^{2}=37.0743, P<0.0001$. b QH of SDU-From Jinan of 2019, compared with that of 2017, $\mathrm{x}^{2}=4.4219, P=0.0355$. From Jinan of 2020 , compared with

bound to affect outpatient visits, including rheumatic patients. The number of outpatient appointments in the rheumatology and immunology department of the three provincial or ministerial hospitals in Jinan had decreased significantly, and the decrease in patients outside of Jinan was more obvious. As a back-up designated hospital for COVID-19 in Shandong Province, Shandong Provincial Hospital had the largest decrease in the number of outpatient appointments of the rheumatology and immunology department. With the gradual control of the epidemic, on 2 March 2020, the 39th day of the first-level response, the number of newly confirmed cases in Shandong province was 0 , with a total of 758 confirmed cases, 6 deaths with mortality rate of $0.79 \%$. The outpatient appointments of rheumatology and immunology department in the three hospitals picked up significantly. It showed that in the face of major infectious diseases, strict quar- that of 2019, $\mathrm{x}^{2}=49.5816, P<0.0001$; compared with that of $2018, x^{2}=38.7641, P<0.0001$; compared with that of 2017 , $\mathrm{x}^{2}=15.8454, P<0.0001$. c AH of SDUTCM -From Jinan of 2020 , compared with that of $2019, x^{2}=112.3106, P<0.0001$; compared with that of 2018, $x^{2}=133.0607, P<0.0001$; compared with that of $2017, x^{2}=113.3615, P<0.0001$. From Jinan of 2019, compared with that of 2017, $x^{2}=6.3306, P=0.0119$. SPH Shandong Provincial Hospital, $Q H$ of SDU Qilu Hospital of Shandong University, AH of SDUTCM Affiliated Hospital of Shandong University of Traditional Chinese Medicine

antine measures with the cooperation of the public can not only control the epidemic in a short time, but also lift the quarantine measures and open general outpatient clinics in hospitals as soon as possible, thus minimizing the impact on other patients.

Among the three provincial or ministerial hospitals in Jinan, the number of outpatient appointments in the rheumatology and immunology department of Affiliated Hospital of SDUTCM decreased the least during the first-level response period in Shandong Province, which indicated that the Chinese public had a high degree of recognition of traditional Chinese medicine in the treatment of rheumatism [18], especially the older patients. The number of patients in the rheumatology and immunology department of Affiliated Hospital of SDUTCM was also the highest in the 50-60 years age group. Traditional Chinese Medicine has a unique therapeutic effect on rheuma- 
Table 4 Comparison of composition ratio of Jinan patients for outpatient appointments among the three provincial and ministerial hospitals from 2017 to 2020

\begin{tabular}{|c|c|c|c|c|c|c|c|c|}
\hline & 2017 & & 2018 & & 2019 & & 2020 & \\
\hline & $x^{2}$ value & $P$ value & $x^{2}$ value & $P$ value & $x^{2}$ value & $P$ value & $x^{2}$ value & $P$ value \\
\hline $\mathrm{SPH}$ to $\mathrm{QH}$ of SDU & 8.9007 & 0.0029 & 6.0165 & 0.0142 & 0.9925 & 0.3191 & 2.7252 & 0.0988 \\
\hline SPH to AH of SDUTCM & 10.4787 & $<0.0001$ & 179.6869 & $<0.0001$ & 173.0594 & $<0.0001$ & 140.9812 & $<0.0001$ \\
\hline $\mathrm{QH}$ of SDU to AH of SDUTCM & 17.7171 & $<0.0001$ & 93.5963 & $<0.0001$ & 177.9506 & $<0.0001$ & 88.29450 & $<0.0001$ \\
\hline
\end{tabular}

Table 5 Analysis of negative binomial regression model

\begin{tabular}{|c|c|c|c|c|c|c|c|c|}
\hline \multirow{2}{*}{\multicolumn{2}{|c|}{ Parameter }} & \multicolumn{2}{|c|}{ Regression coefficient } & \multirow{2}{*}{$\begin{array}{l}\text { Standard } \\
\text { error }\end{array}$} & \multirow{2}{*}{$\begin{array}{l}\text { Wald } \\
\text { chi-square }\end{array}$} & \multirow[t]{2}{*}{$P$ value } & \multicolumn{2}{|c|}{ RR value } \\
\hline & & $\begin{array}{l}\text { Regression } \\
\text { coefficient }\end{array}$ & $\begin{array}{l}95 \% \text { confidence } \\
\text { interval }\end{array}$ & & & & RR value & $\begin{array}{l}95 \% \text { confidence } \\
\text { interval }\end{array}$ \\
\hline \multicolumn{2}{|l|}{ Intercept } & -871.177 & NA & 172.3236 & 25.56 & $<0.0001$ & NA & NA \\
\hline \multicolumn{2}{|l|}{ Year } & 0.4341 & $(0.2661,0.6023)$ & 0.0854 & 25.84 & $<0.0001$ & 1.5436 & $(1.3049,1.8263)$ \\
\hline \multicolumn{2}{|c|}{$\begin{array}{l}\text { Number of days from Chinese New Year's } \\
\text { Eve }\end{array}$} & 0.0267 & $(0.0148,0.0388)$ & 0.0061 & 19.3 & $<0.0001$ & 1.0271 & $(1.0149,1.0396)$ \\
\hline \multicolumn{2}{|l|}{ Week } & -0.3378 & $(-0.4225,0.2542)$ & 0.0428 & 62.41 & $<0.0001$ & 0.7133 & $(0.6554,0.7755)$ \\
\hline \multirow[t]{3}{*}{ Hospitals } & SPH & NA & NA & NA & NA & NA & NA & NA \\
\hline & $\mathrm{QH}$ of SDU & -0.523 & $(-0.8475,-0.1986)$ & 0.1647 & 10.09 & 0.0015 & 0.5927 & $(0.4285,0.8199)$ \\
\hline & AH of SDUTCM & -0.1474 & $(-0.4731,0.1785)$ & 0.1654 & 0.79 & 0.3728 & 0.8629 & $(0.6231,1.1954)$ \\
\hline
\end{tabular}

Table 6 Comparison of actual and predicted values of outpatient appointments in 2020

\begin{tabular}{l|l|l|l|l|}
\hline Hospitals & Actual value & Predicted value & Difference value & Actual value/Predicted value \\
\hline SPH & 1205 & 7998 & -6793 & 0.1507 \\
\hline QH of SDU & 976 & 4738 & -3762 & 0.2060 \\
\hline AH of SDUTCM & 1969 & -4930 & 0.2854 \\
\hline $\begin{array}{l}\text { SPH Shandong Provincial Hospital, QH of SDU Qilu Hospital of Shandong University, AH of SDUTCM Affiliated Hospital of Shandong University of Traditional Chi- } \\
\text { nese Medicine }\end{array}$
\end{tabular}

tism. From more than a thousand years of experience, we have found that many traditional Chinese medicines, such as Tripterygium wilfordii, total glucosides of white peony, and Stephania tetrandra, can effectively treat rheumatism [19, 20]. Acupuncture, massage, expand joint movement and regulated emotions, can also greatly improve the quality of life of arthritis patients [21]. On the other hand, Chinese medicine has played an important role in the prevention and control of COVID-19. In March 2020, the National Health Commission of the People's Republic of China issued the "Guidelines for the Diagnosis and Treatment of Novel Coronavirus Pneumonia (7th edition)", which recommended the use of Qingfei Paidu Decoction (QFPDD) and other traditional Chinese medicine formulations as the treatment of new coronary pneumonia patients. According to reports in China, more than $85 \%$ patients with new coronary pneumonia received Chinese medicine treatment [22].

During the first-level response in Shandong Province, the majority of patients in the rheumatology and immunology department of the three provincial or ministerial hospitals in Jinan were female.
Among them, the proportion of female outpatient appointments in Affiliated Hospital of SDUTCM was higher than that of the same period in previous years; however, the proportion of female outpatient appointments in the three hospitals was higher than $70 \%$ in every year. These showed that the incidence of rheumatism was mainly female. In systemic lupus erythematosus, the prevalence in females is six times higher than in males. The peak age in females is 40-49 years old, which is earlier than 60-69 years old in males [23]. In rheumatoid arthritis, the prevalence is about $0.5-1.0 \%$, the ratio of male to female is about 1:2-3. The onset age of males and females has no significant difference, and the peak age is about 50-69 years old [24]. In our study, proportions of patients aged 30-60 years in every year were $64.13-68.82 \%$ in Shandong Provincial Hospital, 64.05-68.58\% in Qilu Hospital, 68.01-71.81\% in Affiliated Hospital of SDUTCM. It is related to the fact that patients aged 30-60 years have a high incidence of rheumatism and young patients are more willing to use the online appointment system [25].

Rheumatism is a chronic disease that requires long-term drug control. Sudden withdrawal of the 
drug may easily cause disease activity and increase the risk of infection [26]. Rheumatologists from various countries have put forward suggestions: nonCOVID-19 patients are not recommended to interrupt or reduce the immunosuppressive effect, and should ensure continued rheumatism treatment and surveillance $[27,28]$; however, the reality is that hospitals are considered to be the biggest source of infection [29]. In the current COVID-19 pandemic, prolonged and large gatherings of outpatients have significantly increased the chance of infection. At the same time, due to public transportation restrictions, outpatient visits have been affected [30]. Online appointments can optimize medical procedures, significantly shorten patient waiting time, missed diagnosis rates and doctors' punctuality, and reduce cross-infections during the queuing process [31]. But this cannot solve all the problems during the epidemic. Telemedicine may provide basic medical support to the public, including patients with rheumatism, during the COVID-19 outbreak [32]. During the outbreak, telemedicine can also help patients self-isolate, protect other patients, clinicians and the community from contact, reduce the chance of cross-infection in hospitals, eliminate social panic, enhance the public's self-protection ability, correct improper medical treatment behavior, and promote epidemiological screening [33, 34]; however, a lot of issues still need to be further standardized, such as patient privacy protection, medical insurance expense reimbursement, data security, doctor's practice scope and remuneration.

The study indirectly proved the importance of quarantine measures during the COVID-19 epidemic period. Even in a non-outbreak area, strict quarantine measures with the cooperation of the public can not only control the epidemic in a short time, but also lift the quarantine measures and open general outpatient clinics in hospitals as soon as possible, thus minimizing the impact on other patients. The study also reflected the lack of online appointments during the COVID-19 epidemic period. Online appointments can reduce the risk of infection in outpatients, but not completely solve the follow-up problem of rheumatic patients. Telemedicine provides a new solution for both management of rheumatic patients and control of COVID-19; however, further research, standardization and practice are still needed.

Acknowledgements The work was conducted during the Shandong Province's first-level public health emergency response period from 25 January 2020 to 8 March 2020. We thank all the participants in this study for their contribution during this difficult time. We thank the Shandong Shunneng network technology Co., Ltd. for collecting the data. The Shandong Shunneng network technology Co., Ltd. is also grateful to Shandong Provincial Hospital, Qilu Hospital, and Affiliated Hospital of SDUTCM for providing core or unrestricted support.

Funding This work was supported by the National Natural Science Foundation of China (grant number 81671605) and the Medical and Health Technology Development Plan of Shandong Province (grant numbers 2016WS0434 and 2013WS0110). The funders of the study had no role in study design, data collection, data analysis, data interpretation, or writing of the report.

Author Contribution H.S. Sun, Q.R. Yang, and M.Liconceived the study. H.S. Sun, Q.R. Yang, TH Xie, D. Wang, R.H. Liu, and M. Li designed the study. T.H. Xie, D. Wang, X. Wang, and M. Li led data collection. R.H. Liu, and M Li did the statistical analyses. T.H. Xie, D. Wang, and M. Li wrote the first draft of the article. All authors critically reviewed the article and approved the final draft.

\section{Declarations}

Conflict of interest T. Xie, D. Wang, X. Wang, Q. Yang, H. Sun, R. Liu and M. Li declare that they have no competing interests.

Ethical standards Since the study is not included in the biomedical research activities of the "Measures for the Ethical Review of Biomedical Research Involving Humans" formulated by the National Health Commission of P.R. China, it is not suitable for conducting ethical reviews and complying with the principle of informed consent. This retrospective study was performed after consultation with the institutional ethics committee and in accordance with national legal requirements.

\section{References}

1. Zhu N, Zhang D, Wang W, et al. China novel Coronavirus investigating and research team. A novel Coronavirus from patients with pneumonia in China, 2019. N Engl J Med. 2020;382(8):727-33.

2. Coronaviridae Study Group of the International Committee on Taxonomy of Viruses. The species Severe acute respiratory syndrome-related coronavirus: classifying 2019-nCoV and naming it SARS-CoV-2. Nat Microbiol. 2020;5(4):536-44. https://doi.org/10.1038/s41564020-0695-z.

3. World Health Organization. Coronavirus disease (COVID19).. https://www.who.int/emergencies/diseases/novelcoronavirus-2019. Accessed 28 June 2020.

4. Heinz FX, Stiasny K. Profiles of current COVID-19 vaccines. Wien Klin Wochenschr. 2021;16:1-13. https://doi.org/ 10.1007/s00508-021-01835-w. PMID: 33725201; PMCID: PMC7962631.

5. Zhang J, Litvinova M, Liang Y, et al. Changes in contact patterns shape the dynamics of the COVID-19 outbreak in China. Science. 2020;368(6498):1481-6. https://doi.org/ $10.1126 /$ science.abb8001.

6. Civil Protection Department, Government of Italy. Chronology of main steps and legal acts taken by the Italian Governmentfor the containment of theCOVID-19 epidemiological emergency.. http://www.protezionecivile.gov. it/documents/20182/1227694/Summary+of+measures+ taken+against+the+spread+of+C-19/c16459ad-4e52-4e9090f3-c6a2b30c17eb. Accessed 28 June 2020.

7. MottaZanin G, GentileE, Parisi A, Spasiano D. A preliminary evaluation of the public risk perception related to the COVID-19 health emergency in Italy. Int J Environ Res Public Health. 2020;17(9):3024. https://doi.org/10.3390/ ijerph17093024.

8. Department of Economic and Social Affairs Population Dynamics, the United Nations. World population prospects 
2019.. https://population.un.org/wpp/DataSources/. Accessed 28 June 2020.

9. GieseckeJ.Theinvisiblepandemic. Lancet. 2020;395(10238) :e98. https://doi.org/10.1016/S0140-6736(20)31035-7.

10. Viale G, Licata L, Sica L, et al. Personalized risk-benefit ratio adaptation of breast cancer care at the epicenter of COVID 19 outbreak. Oncologist. 2020;25(7):e1013-e20. https:// doi.org/10.1634/theoncologist.2020-0316.

11. Remuzzi A, Remuzzi G. COVID-19 and Italy: what next? Lancet. 2020;395(10231):1225-8. https://doi.org/10.1016/ S0140-6736(20)30627-9.

12. Fagiuoli S, Lorini F, Remuzzi G. Covid-19 Bergamo hospital crisis unit. Adaptations and lessons in the province of Bergamo. N Engl J Med. 2020;382(21):e71. https://doi.org/ 10.1056/NEJMc2011599.

13. Cash R, Patel V. Has COVID-19 subverted global health? Lancet. 2020;395(10238):1687-8. https://doi.org/10.1016/ S0140-6736(20)31089-8.

14. Rosenbaum L. The untold toll-The pandemic's effects on patients without Covid-19. N Engl J Med. 2020;382(24):2368-71. https://doi.org/10.1056/NEJMms 2009984.

15. De Rosa S, Spaccarotella C, Basso C, et al. Reduction of hospitalizations for myocardial infarction in Italy in the COVID-19 era. Eur Heart J. 2020;41(22):2083-8.https://doi. org/10.1093/eurheartj/ehaa409.

16. Caporali R, Favalli E. Managing patients with rheumatic conditions during the covid-19 pandemic. BMJ. 2020;369:m1633. https://doi.org/10.1136/bmj.m1633.

17. Michaud K, Wipfler K, ShawY, et al. Experiences of patients with rheumatic diseases in the United States during early days of the COVID-19 pandemic. ACR Open Rheumatol. 2020;2(6):335-43. https://doi.org/10.1002/acr2.11148.

18. Yuwen Y, Shi NN, Wang LY, Xie YM, Han XJ, Lu AP. Development of clinical practice guidelines in 11 common diseases with Chinese medicine interventions in China. Chin J Integr Med. 2012;18(2):112-9. https://doi.org/10.1007/s11655012-0997-y.

19. Liu Y, Tu S, Gao W, et al. Extracts of tripterygium wilfordii hook $\mathrm{F}$ in the treatment of rheumatoid arthritis: a systemic review and meta-analysis of randomised controlled trials. Evid Based Complement Alternat Med. 2013;2013:410793. https://doi.org/10.1155/2013/410793.

20. Ho L, Lai J. Chinese herbs as immunomodulators and potential disease-modifying antirheumatic drugs in autoimmune disorders. Curr Drug Metab. 2004;5(2):181-92. https://doi.org/10.2174/1389200043489081.

21. Zhang P, Li J, Han Y, Yu XW, Qin L. Traditional Chinese medicine in the treatment of rheumatoid arthritis: ageneral review. Rheumatol Int. 2010;30(6):713-8. https://doi.org/ 10.1007/s00296-010-1370-0.

22. Yang Y, Islam MS, Wang J, Li Y, Chen X. Traditional Chinese medicine in the treatment of patients infected with 2019new Coronavirus (SARS-CoV-2): a review and perspective. Int J Biol Sci. 2020;16(10):1708-17. https://doi.org/10. 7150/ijbs.45538.

23. Rees F, Doherty M, Grainge M, Davenport G, Lanyon P, Zhang W. The incidence and prevalence of systemic lupus erythematosus in the UK, 1999-2012. Ann Rheum Dis. 2016;75(1):136-41. https://doi.org/10. 1136/annrheumdis-2014-206334.

24. Kato E, Sawada T, Tahara K, et al. The age at onset of rheumatoid arthritis is increasing in Japan: a nationwide database study. Int J Rheum Dis. 2017;20(7):839-45. https://doi.org/10.1111/1756-185X.12998.

25. ZhangX, Yu P, Yan J, Spil ITAM. Using diffusion of innovation theory to understand the factors impacting patient acceptance and use of consumer e-health innovations: a case study in a primary care clinic. BMC Health Serv Res. 2015;15:71. https:// doi.org/10.1186/s12913-015-0726-2.

26. Smolen J, Breedveld F, Burmester G, et al. Treating rheumatoid arthritis to target: 2014 update of the recommendations of an international task force. Ann Rheum Dis. 2016;75(1):3-15. https://doi.org/10.1136/annrheumdis2015-207524.

27. Schulze-Koops H, Specker C, Iking-Konert C, Holle J, Moosig F, Krueger K. Preliminary recommendations of the German Society of Rheumatology (DGRh eV) for the management of patients with inflammatory rheumatic diseases during the SARS-CoV-2/COVID-19 pandemic. Ann Rheum Dis. 2020;79(6):840-2. https://doi.org/10.1136/ annrheumdis-2020-217628.

28. Mikuls TR, JohnsonSR, FraenkelL, etal. American College of Rheumatology guidance for the management of rheumatic disease in adult patients during the COVID-19 pandemic: version 2. Arthritis Rheumatol. 2020; https://doi.org/10. $1002 /$ art.41437.

29. Heymann D. Data sharing and outbreaks: best practice exemplified. Lancet. 2020;395(10223):469-70. https://doi. org/10.1016/S0140-6736(20)30184-7.

30. Zhang H. Early lessons from the frontline of the $2019-\mathrm{nCoV}$ outbreak. Lancet. 2020;395(10225):687. https://doi.org/ 10.1016/S0140-6736(20)30356-1.

31. Habibi MRM, Mohammadabadi F, Tabesh H, Vakili-Arki H, Abu-Hanna A, Eslami S. Effect of an online appointment scheduling system on evaluation metrics of outpatient scheduling system: a before-after multicenterstudy. J Med Syst. 2019;43(8):281. https://doi.org/10.1007/s10916-0191383-5.

32. Webster P. Virtual health care in the era of COVID-19. Lancet. 2020;395(10231):1180-1. https://doi.org/10.1016/ S0140-6736(20)30818-7.

33. Bakshi SS, Kalidoss VK. Patient concern inventory: an effective tool for easing telemedicine during the COVID-19 pandemic. Wien Klin Wochenschr. 2021;14:1-2. https:// doi.org/10.1007/s00508-021-01860-9.

34. Gong K, Xu Z, Cai Z, Chen Y, Wang Z. Internet hospitals help prevent and control the epidemic of COVID-19 in China: multicenter user profiling study. J Med Internet Res. 2020;22(4):e18908. https://doi.org/10.2196/18908.

Publisher's Note Springer Nature remains neutral with regard to jurisdictional claims in published maps and institutional affiliations. 